\title{
Die Impulsoszillometrie in der Schweregraddiagnostik obstruktiver Lungenerkrankungen ${ }^{1,2,3}$
}

\author{
Impulse Oscillometry in the Diagnosis of the Severity of Obstructive Pulmonary \\ Disease
}

Autoren

Institute
J. Winkler ${ }^{1}$, A. Hagert-Winkler ${ }^{2}$, H. Wirtz ${ }^{3}$, J. Schauer ${ }^{3}$, T. Kahn², G. Hoheisel ${ }^{1}$

Fachpraxen für Pneumologie und Allergologie, Leipzig

Klinik und Poliklinik für Diagnostische Radiologie, Klinikum der Universität Leipzig AöR

Department Innere Medizin, Abteilung Pneumologie, Universitätsklinikum Leipzig AöR eingereicht 1.9.2008

akzeptiert nach Revision

27. 1.2009

Bibliografie

DOI $10.1055 / \mathrm{s}-0028-1119698$

Online-Publikation: 25. 3. 2009

Pneumologie 2009; 63: 266-275

(c) Georg Thieme Verlag KG

Stuttgart · New York

ISSN 0934-8387

\section{Korrespondenzadresse}

Dr. med. Jörg Winkler

Fachpraxis für Pneumologie

und Allergologie

Kreuzstr. 2a

04103 Leipzig

lungewinkler@aol.com

\section{Zusammenfassung}

Die Impulsoszillometrie (IOS) ist eine computergestützte Methode zur Messung komplexer mechanischer Eigenschaften der Atemwege. Ziel dieser Studie war die IOS in der Diagnose obstruktiver Atemwegserkrankungen im Hinblick auf die praktische Anwendbarkeit vergleichend zu den Standardverfahren Spirometrie, Pneumotachygrafie und Bodyplethysmografie zu evaluieren. Retrospektiv wurden 244 Patienten (Alter 61,5 $\pm 13,6 \mathrm{~J}$; $61 \%$ männlich) mit Asthma bronchiale $(n=65)$ und chronisch obstruktiver Lungenerkrankung (chronic obstructive pulmonary disease, COPD) ( $n=179$ ) untersucht. Mittels Bodyplethysmografie (SRtot $>120 \%$ SW) wurde bei $94 \%$ der Patienten eine Obstruktion diagnostiziert, mittels Pneumotachografie (FEV1 < 80\%SW) bei 78\% und mittels IOS je nach Parameter bei $87-94 \%$. Leicht- und mittelgradige Obstruktionen wurden durch alle IOS-Parameter außer R20 erfasst, schwere Obstruktionen jedoch besser durch die Frequenzabhängigkeit der Resistance FDR und die Reactance bei $5 \mathrm{~Hz}$ X5. Die Parameter R5, X5 und Zrs zeigten jedoch eine Tendenz zur Unterschätzung des Obstruktionsgrades. Eine bronchiale Obstruktion kann durch die IOS insgesamt sicher diagnostiziert werden, sofern bei schwerer Obstruktion komplexe IOS-Parameter wie FDR und X5 mit einbezogen werden. Die Parameter der Standardverfahren (Bodyplethysmografie und Pneumotachografie) zeigten aufgrund unterschiedlicher Messprinzipien insgesamt geringe statistische Korrelationen sowohl zur IOS, als auch im Vergleich untereinander. Zusammenfassend ist die IOS nach den Ergebnissen der Untersuchung eine patientenschonende und wenig kooperationsabhängige Methode zur Evaluierung obstruktiver Atemwegserkrankungen, welche bisherige Standardmethoden sinnvoll ergänzt und sich darüber hinaus als sensitives Screeninginstrument zur frühzeitigen Erkennung einer Bronchialobstruktion eignet.

\section{Abstract}

Impulse oscillometry (IOS) is a computer-supported method for the measurement of complex mechanical airway characteristics. The aim of this study was to evaluate the applicability of IOS in the diagnosis of obstructive airway diseases in comparison to the standard methods of spirometry, pneumotachymetry, and bodyplethysmography. 244 patients (age $61.5 \pm 13.6$ years; $61 \%$ men) with bronchial asthma $(n=65)$ and chronic obstructive pulmonary disease (COPD) $(n=179)$ were retrospectively analysed. By means of body plethysmography (Srtot < 120\%pred) an obstruction was diagnosed in $94 \%$, by pneumotachymetry (FEV1 < $80 \%$ pred) in $78 \%$, and by IOS depending on the parameter chosen in $87-94 \%$ of patients. Mild and medium obstructions could be identified by means of all IOS parameters except for R20: severe obstructions, however, were better detected by means of the frequency-dependant resistance FDR and reactance at $5 \mathrm{~Hz}, \mathrm{X} 5$. The parameters R5, X5, and Zrs, however, showed a tendency to underestimate the degree of obstruction. Bronchial obstruction can reliably be diagnosed by IOS when, in cases of severe obstruction, more complex parameters like FDR and X5 are included. The parameters of the standard methods (body plethysmography and pneumotachymetry) show relatively low correlations due to the different measurement methods not only in comparison to IOS but also to one another. In summary, IOS is a convenient method for patients with a low dependency on cooperation for the evaluation of obstructive airway diseases complementary to the established standard methods and, in addition, is useful as a sensitive screening tool for the early detection of bronchial obstruction.

\footnotetext{
${ }^{1}$ Jörg Winkler und Anne Hagert-Winkler haben zu gleichen Teilen zu der Arbeit beigetragen.

2 Teile der Arbeit sind Gegenstand der Dissertationsschrift von Anne Hagert-Winkler.

${ }^{3}$ Herrn Professor Schauer, unserem klinischen Lehrer, zum 70. Geburtstag gewidmet.
} 


\section{Einleitung}

\section{$\nabla$}

Zur Messung des Strömungswiderstandes, des Einsekundenvolumens (FEV1) und weiterer Parameter einer Atemwegsobstruktion stehen heute verschiedene Methoden zur Verfügung [1-9]. Eine modernen Ansprüchen genügende Lungenfunktionsdiagnostik sollte bei vertretbarem technischem und finanziellem Aufwand die wesentlichen Komponenten der Obstruktion bei geringer Kooperationsabhängigkeit und Patientenbelastung erfassen und im Idealfall ortsvariabel (z.B. am Arbeitsplatz) einsetzbar sein. Ziel dieser Arbeit ist die bei Erwachsenen mit verschiedenen Schweregraden einer Atemwegsobstruktion mittels IOS ermittelten Werte mit denen der Bodyplethysmografie und Spirometrie/ Pneumotachografie zu vergleichen. Darüber hinaus sollte geprüft werden, welche IOS-Parameter für die Erfassung und Schweregradeinstufung bronchialer Obstruktionen bei Erwachsenen geeignet sind und welche Konsequenzen sich daraus für den praktischen Einsatz dieser Methode ableiten lassen.

\section{Messverfahren und Problemstellung \\ $\nabla$}

Mittels Spirometrie bzw. Pneumotachografie lässt sich eine Obstruktion bei forcierter Atmung durch Messung des Einsekundenvolumens (FEV1), der relativen Einsekundenkapazität (Tiffeneau-Index, FEV1\%VCin) sowie exspiratorischer Flusswerte (MEF) quantifizieren. Das von der Patientenmitarbeit abhängige FEV1 zeigt bei chronisch obstruktiver Lungenerkrankung (COPD) in der Regel eine geringe, beim Asthma bronchiale eine größere Variabilität [10-14]. Bei der COPD kommt es durch Elastizitätsverlust zur exspiratorischen bronchialen Kollapsneigung mit Flussbegrenzung bis hin zum „Emphysemknick“ [15-16]. Sehr leichte Obstruktionen, z. B. beim Asthma bronchiale, können teils durch forcierte Atemmanöver muskulär kompensiert und somit unterschätzt werden. Darüber hinaus ist eine Schädigung der peripheren Atemwege oft nur eingeschränkt erfassbar („quiet zone“), sodass die Frühdiagnose erschwert wird [16-20]. Die mit der Pneumotachografie zusätzlich erfassten mittleren exspiratorischen Flusswerte (MEF bei 50 und 25\% der Vitalkapazität) gelten als weniger mitarbeitsabhängig und liefern Hinweise auf eine periphere Atemwegsobstruktion („small airways disease“) [19-26].

Die Ganzkörperplethysmografie (Bodyplethysmografie) gilt als Goldstandard und erlaubt bei Ruheatmung u. a. die Bestimmung des intrathorakalen Gasvolumens (ITGV), der funktionellen Residualkapazität (FRC), des Residualvolumens (RV), des Atemwegswiderstands (z.B. als Rtot) und der spezifischen Resistance (SRtot) [13,21,25,27-32]. Nachteile sind jedoch der apparative Aufwand, hohe Kosten und die fehlende Mobilität des Messsystems [27,32-34].

Die Methodik der Impulsoszillometrie (IOS), bei welcher durch einen externen Generator erzeugte sinusförmige Schwingungen (Oszillationen) der Ruheatmung des Patienten überlagert werden, stellt eine Weiterentwicklung der klassischen Oszillometrie dar. Die Antwort des respiratorischen Systems wird durch Registrierung der am Mundstück entstehenden Wechselströmung sowie des Wechseldrucks analysiert. Vorteile sind der geringe apparative Aufwand und die weitgehende Unabhängigkeit der Messung von der Mitarbeit des Patienten [32,35]. Periphere und zentrale Obstruktionen sind durch spezifische Parameter bei verschiedenen Messfrequenzen charakterisierbar [36]. Die IOS erlaubt ferner eine fortlaufende Widerstandsbestimmung ohne
Tab. 1 Ein- und Ausschlusskriterien.

$\begin{array}{lc}\text { Einschlusskriterien } & \text { Ausschlusskriterien } \\ \begin{array}{l}\text { 1. schriftlicher ärztlicher Unter- } \\ \text { suchungsauftrag }\end{array} & \begin{array}{c}\text { 1. Verletzung der Untersuchungs- } \\ \text { reihenfolge }\end{array} \\ \begin{array}{l}\text { 2. ausreichende Kooperation } \\ \text { 3. stabile klinische Situation ohne } \\ \text { Atemwegsinfekt }\end{array} & \begin{array}{c}\text { 2. Nichteinhaltung der Medika- } \\ \text { mentenpause vor der Unter- } \\ \text { suchung }\end{array} \\ \begin{array}{ll}\text { 4. obstruktive Normabweichun- } \\ \text { gen in der Basis-Lungenfunk- }\end{array} & \begin{array}{l}\text { 3. Nichteinhaltung der Wartezeit } \\ \text { tionsdiagnostik }\end{array} \\ \begin{array}{ll}\text { 5. keine bronchospasmolytische } \\ \text { Medikation in den letzten 6 h }\end{array} & \begin{array}{l}\text { molytischen Medikation } \\ \text { (kurzwirksame Beta-2-Mime- }\end{array} \\ \text { tika), 12 h (langwirksame } & \text { 30' nach Anticholinergika) } \\ \text { Beta-2- Mimetika) } & \text { holungsmessungen }\end{array}$

Tab. 2 Patientencharakteristika der Gesamtgruppe $(n=244)$.

\begin{tabular}{|c|c|c|c|}
\hline $\begin{array}{l}\text { Parameter } \\
\text { MW } \pm \text { s }\end{array}$ & $\begin{array}{l}\text { Gesamt- } \\
\text { gruppe }\end{array}$ & $\begin{array}{l}\text { Asthma } \\
\text { bronchiale }\end{array}$ & COPD \\
\hline$n(\%)$ & 244 & $65(27 \%)$ & $179(73 \%) \mathrm{ns}$ \\
\hline n (\%) männlich & $150(61 \%)$ & $30(46 \%)$ & 120 (67\%) ns \\
\hline Alter (J) & $61,5 \pm 13,6$ & $52 \pm 13,3$ & $64,9 \pm 12,0^{*}$ \\
\hline Größe (cm) & $169 \pm 8,5$ & $169 \pm 8,3$ & $169 \pm 8,5$ ns \\
\hline Gewicht (kg) & $75,9 \pm 16,1$ & $77,5 \pm 14,2$ & $75,4 \pm 16,7 \mathrm{~ns}$ \\
\hline
\end{tabular}

die aktive Mitarbeit des Patienten, was sich besonders nützlich auch bei jüngeren Kindern erweist [1,37-50].

Ziel dieser Arbeit ist die Beurteilung von Sensitivität und Schweregradeinstufung bronchialer Obstruktionen bei Erwachsenen mittels IOS im Vergleich zu den Standardverfahren Bodyplethysmografie und Spirometrie/Pneumotachografie.

\section{Patienten und Methoden}

Die Lungenfunktionsdaten von 244 Patienten, die im Rahmen de Routinediagnostik in der Abteilung Pneumologie, Medizinische Klinik und Poliklinik I, Universitätsklinikum Leipzig, zwischen Oktober 1995 und April 2000 untersucht worden waren, wurden retrospektiv analysiert. Die Ein- und Ausschlusskriterien sind in - Tab. 1, wesentliche Patientencharakteristika in $\bullet$ Tab. 2 dargestellt.

\section{Untersuchungsablauf und Bronchospasmolysetestung} Nach Aufklärung und Einwilligung des Patienten wurden durchgeführt:

1. IOS-Messung (Basiswerte)

2. Bodyplethysmografie mit integrierter Pneumotachografie/ Spirometrie

3. Verabreichung von inhalativen Bronchospasmolytika

4. Wartezeit abhängig von der Substanz in Nähe des Untersuchungsraumes

5. IOS-Folgemessung

6. Bodyplethysmografie mit integrierter Pneumotachografie/ Spirometrie nach Lyse

Die Messungen erfolgten mittels IOS (MasterScreen IOS, Cardinal Health, Jaeger GmbH, D-97204 Höchberg) und Ganzkörperplethysmografie/Pneumotachografie (Masterlab, volumenkonstantes System, Cardinal Health, Jaeger GmbH, D-97204 Höchberg) gemäß standardisierter Empfehlungen [51 -56]. Die abteilungs- 


\begin{tabular}{llll|}
$\begin{array}{l}\text { Schweregrad/ } \\
\text { Parameter }\end{array}$ & $\begin{array}{l}\text { Obstruktion bei forcierter } \\
\text { Atmung, sofern FEV1\% FVC }<70\end{array}$ & $\begin{array}{l}\text { Obstruktion bei Ruhe- } \\
\text { atmung SRtot \%SW }\end{array}$ & $\begin{array}{l}\text { Lungenüberblähung } \\
\text { RV \%TLC }\end{array}$ \\
\hline leichtgradig & FEV1\%SW $\geq 80$ & $>120$ bis $\leq 200$ & $>45$ bis $\leq 60$ \\
\hline mittelgradig & FEV1 $\%$ SW $<80 \geq 50$ & $>200$ bis $\leq 400$ & $>60$ bis $\leq 70$ \\
\hline schwergradig & FEV1\%SW $<50$ & $>400$ & $>70$ \\
\hline
\end{tabular}

Tab. 3 Funktionsdiagnostische Grenzwerte zur Schweregradeinteilung von Obstruktion und Lungenüberblähung.

\begin{tabular}{|c|c|c|c|c|}
\hline $\begin{array}{l}\text { Parameter/statistischer } \\
\text { Gruppenwert }\end{array}$ & Mittelwert & $\begin{array}{l}\text { Standard- } \\
\text { abweichung }\end{array}$ & $\begin{array}{l}\text { Variations- } \\
\text { koeffizient in \% }\end{array}$ & $\begin{array}{l}\text { Minimum/ } \\
\text { Maximum }\end{array}$ \\
\hline Rtot (kPa/l/s) & 0,62 & 0,27 & 43 & $0,21 / 1,63$ \\
\hline Rtot \%SW & 205 & 89,5 & 43 & $71 / 544$ \\
\hline SRtot \%SW & 249 & 129,7 & 52 & $82 / 774$ \\
\hline FEV1 (I) & 1,76 & 0,69 & 39 & $0,53 / 4,43$ \\
\hline FEV $1 \% S W$ & 63 & 20,4 & 32 & $22 / 113$ \\
\hline FEV1 \%VCin & 61 & 12,6 & 21 & $30 / 88$ \\
\hline PEF \%SW & 58 & 21,4 & 37 & $11 / 113$ \\
\hline MEF50\%SW & 31 & 19,1 & 62 & $7 / 102$ \\
\hline MEF25 \%SW & 26 & 16,4 & 63 & $2 / 95$ \\
\hline TLC \%SW & 102 & 14,2 & 14 & $70 / 143$ \\
\hline RV \%TLC & 53 & 10,4 & 20 & $23 / 76$ \\
\hline VCin \%SW & 79 & 16,5 & 21 & $35 / 128$ \\
\hline
\end{tabular}

Tab. 4 Gemessene Standardparameter der Lungenfunktion (Bodyplethysmografie und Pneumotachografie) in der Gesamtgruppe ( $\mathrm{n}=244)$. intern angewandte Definition der Obstruktionsschweregrade ist in Tab. 3 dargestellt $[21,57]$. Zur besseren Vergleichbarkeit mit den Resistancewerten der IOS-Methode erfolgte die Schweregradeinteilung anhand des Leitparameters SRtot (spezifische Resistance), welcher die COPD-typische Lungenüberblähung mit einbezieht $[28,58]$. Auf eine Darstellung der Ergebnisse der Bronchospasmolysetestung (Untersuchungsschritte 3-6) wurde verzichtet.

Da die impulsozillometrischen Resistancewerte, wie oben ausgeführt, besonders bei peripherer Obstruktion eine deutliche Frequenzabhängigkeit mit höheren Werten insbesondere bei niedrigen Frequenzen zeigen, wurde zur Quantifizierung dieser Frequenzabhängigkeit der Parameter „Frequenzabhängigkeit der Resistance“ (FDR) absolut und relativ nach folgender Vorschrift berechnet [59-62]:

absolute Frequenzabhängigkeit:

$\mathrm{FDRa}=\mathrm{R} 5-\mathrm{R} 20$

standardisierte (relative) Frequenzabhängigkeit:

$\mathrm{FDR}=(\mathrm{R} 5-\mathrm{R} 20) / \mathrm{R} 20$

\section{Statistische Datenanalyse}

Die Ergebnisse sind als Mittelwerte $(\mathrm{MW}) \pm$ Standardabweichung (s) wiedergegeben. Die statistische Kalkulation und Erstellung von Grafiken erfolgten mit Hilfe des Computerprogramms SPSS ${ }^{\circledR}$ (Version 10.0, SPSS ${ }^{\circledR}$, D-Erkrath) und Excel ${ }^{\circledR}$ Version 8 (Micro$\left.\operatorname{soft}^{\circledR}\right)$. Zur Prüfung individueller Differenzen abhängiger Stichproben wurde der parameterfreie Wilcoxon-Test und für Vergleiche zwischen unabhängigen Gruppen der Mann-Whitney-U-Test eingesetzt. Der Korrelationskoeffizient nach Pearson wurde als Maß für statistische Zusammenhänge verwendet und gegen 0 auf statistische Signifikanz geprüft. Befunde mit einem p-Wert $\leq 0.05$ wurden als signifikant bezeichnet.

\section{Ergebnisse}

$\nabla$

Das Durchschnittsalter von Patienten mit COPD war signifikant höher im Vergleich zu solchen mit Asthma bronchiale, die übrigen Parameter waren vergleichbar. Bei den COPD-Patienten war der Anteil männlicher Patienten erhöht im Vergleich zu einem ausgeglichenen Geschlechterverhältnis bei Asthma bronchiale (৫ Tab. 2).

\section{Quantifizierung der Obstruktion mittels}

Bodyplethysmografie/Pneumotachygrafie

Es zeigte sich im Gruppenmittel eine mittelgradige Erhöhung des Atemwegswiderstandes bei Ruheatmung ( Tab.4). Das erhöhte Residualvolumen spricht bei eingeschränkter Vitalkapazität und normaler Totalkapazität für eine leichtgradige relative Lungenüberblähung. FEV1\%SW und FEV1\%VCin waren erniedrigt. Gemäß den Kriterien der Schweregradeinteilung ( Tab.3) fand sich eine leicht- bis mittelgradige obstruktive Ventilationsstörung mit leichter relativer Lungenüberblähung. Über dem Sollgrenzwert von $120 \%$ liegende Werte für die spezifische Resistance fanden sich bei 229 Patienten (94\%). Unter dem Sollgrenzwert für FEV1\%VCin von 70\% lagen 182 Patienten (75\%). Ein absolutes FEV1 von < 80\%SW hatten 190 Patienten (78\%). 173 Patienten (71\%) hatten pathologische Werte in beiden Leitparametern (SRtot und FEV1\%VCin), bei 65 Patienten (23\%) erfolgte die Einstufung als „obstruktiv“ nur nach einem der beiden Parameter (SRtot $n=56$, FEV1 \%VCin n = 9). SRtot zeigte trotz größerer Streuung eine stärkere prozentuale Normabweichung gegenüber Rtot ( $p<0,001)$. Zwischen den Leitparametern der Bodyplethysmografie (SRtot \%SW) und der Pneumotachografie (FEV1) zeigte sich eine schwache statistische Korrelation $(r=-0,49, p<0,01)$.

\section{Quantifizierung der Obstruktion mittels IOS}

Die Resistancewerte waren besonders bei $5 \mathrm{~Hz}$ (202\%SW) gegenüber den Sollwerten deutlich erhöht, bei $20 \mathrm{~Hz}$ jedoch nur grenzwertig (140\%SW) ( Tab. 5). Die Reactance X lag bei $5 \mathrm{~Hz}$ deutlich im pathologischen Negativbereich. Die Impedanz (Zrs \%SW) zeigte eine Normwertüberschreitung von $223 \%$, die über der von R5 und R20 lag ( $\mathrm{p}<0,001)$.

Im Vergleich zwischen Widerstandsparametern der Bodyplethysmografie und der IOS war die größte Normwertabweichung für SRtot feststellbar ( $p<0,05$ vs. Z, übrige $p<0,001$ ). Die Sollwertabweichung von R5 war der von Rtot vergleichbar. 


\begin{tabular}{|c|c|c|c|c|c|c|}
\hline $\begin{array}{l}\text { Parameter/statisti- } \\
\text { scher Gruppenwert }\end{array}$ & $\begin{array}{l}\text { Sollwerte ( } \pm \text { s bzw. } \\
\leq \text { Grenzwerte) }\end{array}$ & $\begin{array}{l}\text { Mittel- } \\
\text { wert }\end{array}$ & $\begin{array}{l}\text { Standard- } \\
\text { abweichung }\end{array}$ & $\begin{array}{l}\text { Variationsko- } \\
\text { effizient in \% }\end{array}$ & $\begin{array}{l}\text { Minimum/ } \\
\text { Maximum }\end{array}$ & \multirow{11}{*}{$\begin{array}{l}\text { Tab. } 5 \text { IOS-Parameter in der } \\
\text { Gesamtgruppe ( } n=244 \text {, Über- } \\
\text { schreitungen der Sollwerte } \\
\text { fettgedruckt). }\end{array}$} \\
\hline $\mathrm{R} 5(\mathrm{kPa} / \mathrm{l} / \mathrm{s})$ & $0,34 \pm 0,05^{*}$ & 0,67 & 0,25 & 37 & $0,27 / 1,60$ & \\
\hline R5 \% SW & $\leq 130-150^{* *}$ & 202 & 74,5 & 37 & $81 / 454$ & \\
\hline $\mathrm{R} 20(\mathrm{kPa} / \mathrm{l} / \mathrm{s})$ & $0,29 \pm 0,04^{*}$ & 0,40 & 0,13 & 32 & $0,14 / 1,05$ & \\
\hline R20\% SW & $\leq 130-150^{* *}$ & 140 & 45,1 & 32 & $59 / 393$ & \\
\hline FDRa & $0,06 \pm 0,03^{*}$ & 0,27 & 0,17 & 63 & $-0,06 / 0,97$ & \\
\hline FDR & $0,16 \pm 0,02^{*}$ & 0,68 & 0,37 & 54 & $-0,11 / 2,47$ & \\
\hline $\mathrm{X} 5(\mathrm{kPa} / \mathrm{l} / \mathrm{s})$ & $-0,04 \pm 0,04^{*}$ & $-0,31$ & 0,21 & 68 & $-1,41 / 0,54$ & \\
\hline Fres $(\mathrm{Hz})$ & $12,4 \pm 5,2^{*}$ & 23,8 & 6,64 & 28 & $7,5 / 39,7$ & \\
\hline Zrs (kPa/l/s) & $0,34 \pm 0,05^{*}$ & 0,75 & 0,30 & 40 & $0,27 / 2,13$ & \\
\hline Zrs \% SW & $\leq 130-150^{* *}$ & 223 & 87,9 & 40 & $86 / 498$ & \\
\hline
\end{tabular}

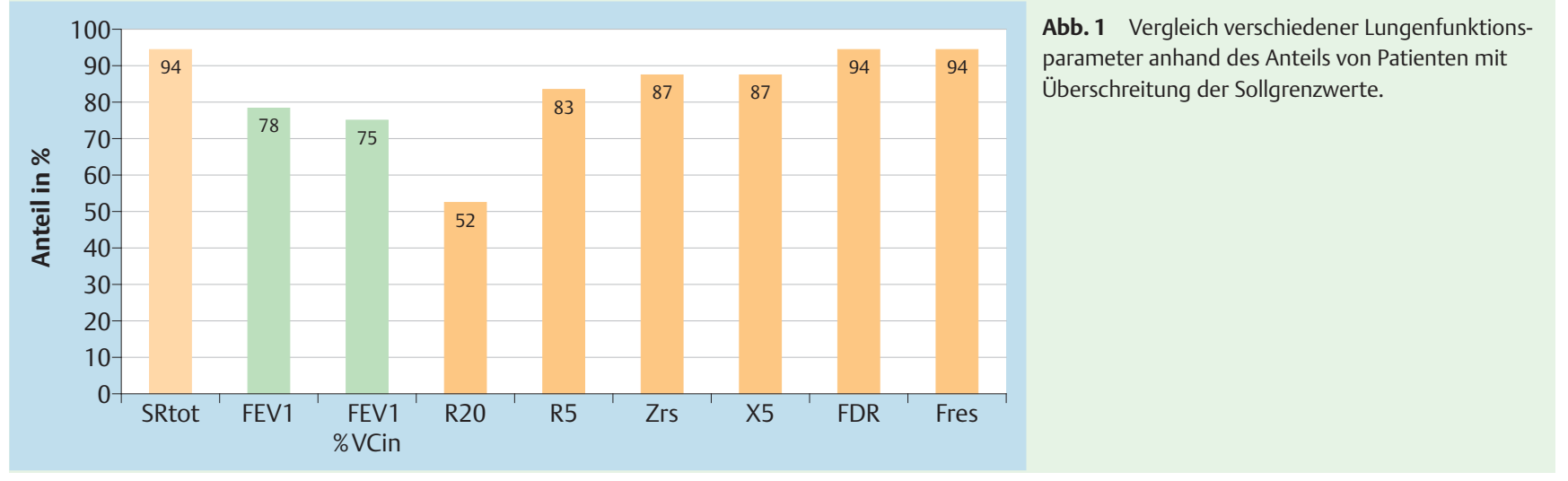

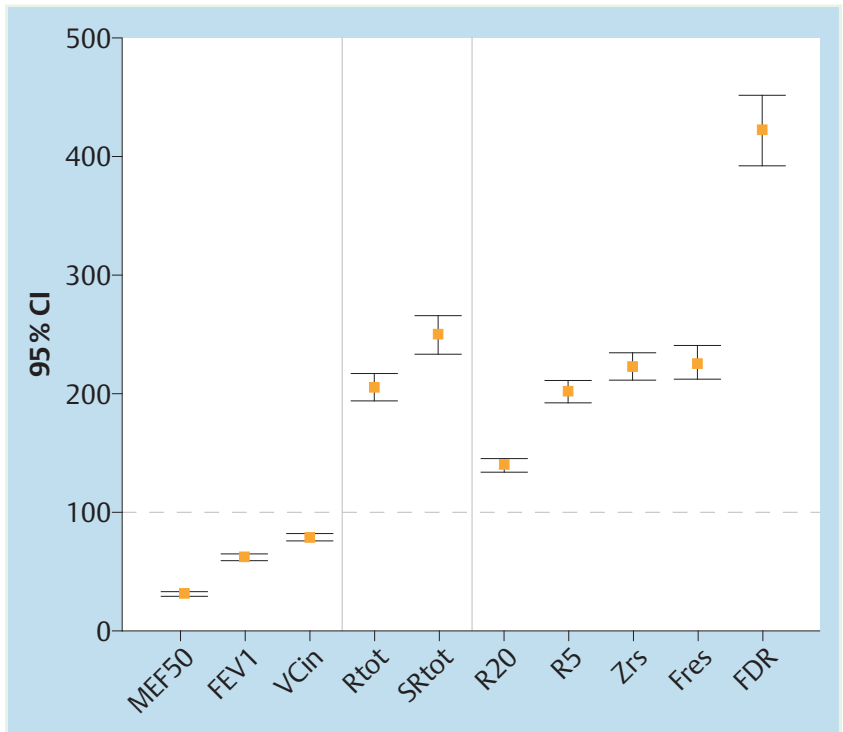

Abb. 2 Vergleich verschiedener Lungenfunktionsparameter anhand der prozentualen Abweichungen vom Sollmittelwert, Gegenüberstellung von Pneumotachografie, Bodyplethysmografie und Impulsoszillometrie.

Die über den Frequenzbereich 5 bis $20 \mathrm{~Hz}$ erfassbare Abhängigkeit der Resistance, definiert als FDR-Maßzahl, lag mit 0,68 deutlich über dem erwarteten Wert. Die Resonanzfrequenz, bei der die Reactance X den Wert 0 erreicht, war im Mittel um $10-16 \mathrm{~Hz}$ höher als in Referenzkollektiven beschrieben [63-68]. Die Sollwerte um mehr als 30\% überschreitende Beträge fanden sich für Zrs bei $87 \%$, für R5 bei $83 \%$ und für R20 bei $52 \%$ der Patienten $(\bullet$ Abb. 1$)$.
Die Reactance lag bei $87 \%$ der Messungen unterhalb des Sollgrenzwertes von $-0,12 \mathrm{kPa} / \mathrm{l} / \mathrm{s}$. Die Resonanzfrequenz lag bei 230 Patienten (94\%) oberhalb des in der Literatur angegebenen mittleren Sollwertes von $12 \mathrm{~Hz}$. Der in der Literatur angegebene Sollgrenzwert $(\mathrm{MW}+2 \mathrm{~s})$ der absoluten Frequenzabhängigkeit (FDRa) von 0,12 [62] wurde bei 232 Patienten (95\%) überschritten. Die standardisierte (relative) Frequenzabhängigkeit der Resistance FDR lag bei 94\% der Patienten oberhalb des berechneten Grenzwertes von 0,2 standardisierten Einheiten ( $\bullet$ Abb. 2).

Die Korrelation zwischen den Parametern der Standardmethodik und der IOS ist relativ schwach $(\square$ Tab. 6 und $\bullet$ Abb. 3). Die IOS-Parameter korrelieren deutlicher zu Widerstandsparametern der Bodyplethysmografie (SRtot, Rtot) als zu den Flussparametern der Pneumotachografie. Für die komplexeren IOSParameter X5, Fres und Zrs ist eine Korrelation zu den forcierten Flusswerten (FEV1, PEF, MEF) und zur Lungenüberblähung (RV \%TLC) feststellbar. Für R20 liegen keine wesentlichen Korrelationen zu Parametern der Standardlungenfunktion vor, während R5 zur Resistance Rtot korreliert. Trotz der fehlenden Korrelation der Absolutwerte von R20 und der nur geringen von R5 zu den forcierten Flusswerten und dem Residualvolumen korreliert die Frequenzabhängigkeit von R5 und R20 (FDR) mit allen Vergleichsparametern der Standardlungenfunktion und ist damit den komplexeren IOS-Parametern X5, Fres, Zrs vergleichbar. Von den Parametern der statischen Lungenvolumina bzw. der Lungenüberblähung wurde nur RV \%TLC dargestellt, da sich für die übrigen statischen Volumina noch schwächere statistische Zusammenhänge ergaben (z.B. VCin zu Fres, r = -0,22, p<0,01). 


\begin{tabular}{|c|c|c|c|c|c|c|c|}
\hline $\begin{array}{l}\text { Parameter| } \\
\text { Korrelationen zu }\end{array}$ & FEV1(I) & PEF \% SW & MEF50\% SW & Rtot \% SW & SRtot \% SW & RV \% TLC & $\begin{array}{l}\text { Tab. } 6 \text { Korrelationskoeffizien- } \\
\text { ten nach Pearson zwischen }\end{array}$ \\
\hline R5 (kPa/l/s) & $-0,28^{* *}$ & $-0,27^{* *}$ & $-0,17^{* *}$ & $0,48^{* *}$ & $0,37^{* *}$ & $0,22^{*}$ & $\begin{array}{l}\text { Parametern der Standard- } \\
\text { methodik und IOS }(n=244) \text {. }\end{array}$ \\
\hline $\mathrm{R} 20(\mathrm{kPa} / \mathrm{l} / \mathrm{s})$ & 0,09 & $-0,10$ & 0,04 & 0,20 & 0,07 & $-0,01$ & \\
\hline FDR & $-0,36^{* *}$ & $-0,34^{* *}$ & $-0,36^{* *}$ & $0,52^{* *}$ & $0,54^{* *}$ & $0,42^{* *}$ & \\
\hline $\mathrm{X} 5(\mathrm{kPa} / \mathrm{l} / \mathrm{s})$ & $0,38 * *$ & $0,32 * *$ & $0,27^{* *}$ & $-0,59^{* *}$ & $-0,52^{* *}$ & $-0,37^{* *}$ & \\
\hline Fres $(\mathrm{Hz})$ & $-0,41^{*}$ & $-0,34^{* *}$ & $-0,34^{* *}$ & $0,53^{* *}$ & $0,51^{* *}$ & $0,44^{* *}$ & \\
\hline $\mathrm{Zrs}(\mathrm{kPa} / \mathrm{l} / \mathrm{s})$ & $-0,33^{* *}$ & $-0,32^{* *}$ & $-0,23^{* *}$ & $0,57^{* *}$ & $0,48^{* *}$ & $0,29 * *$ & \\
\hline
\end{tabular}
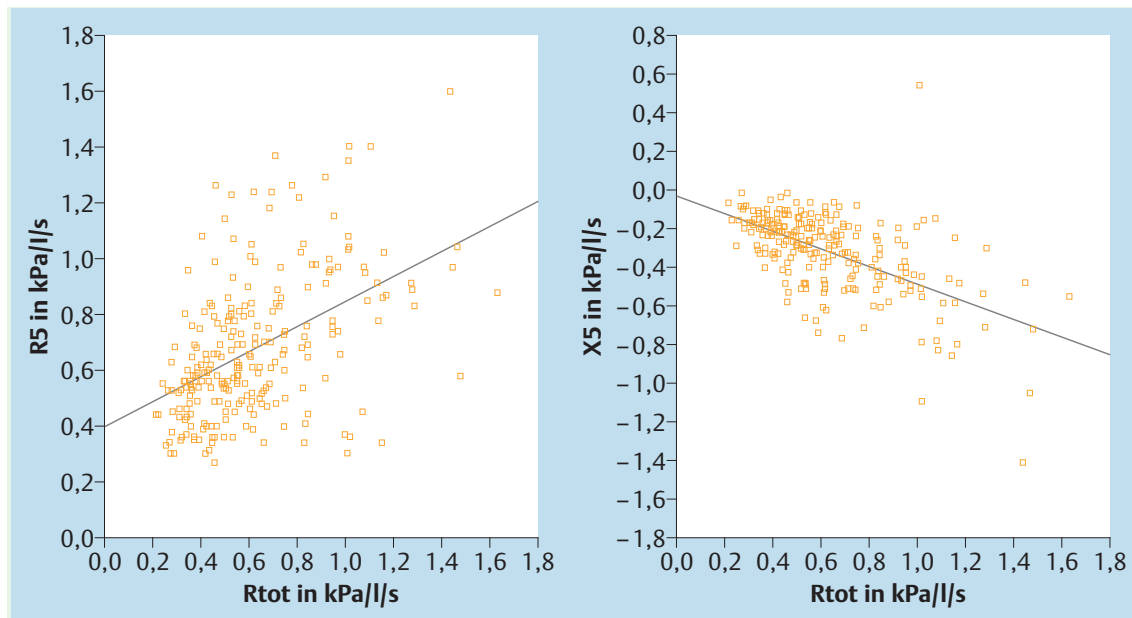

Abb. 3 Korrelationen zwischen ausgewählten Parametern der Standardmethodik und der Impulsoszillometrie.
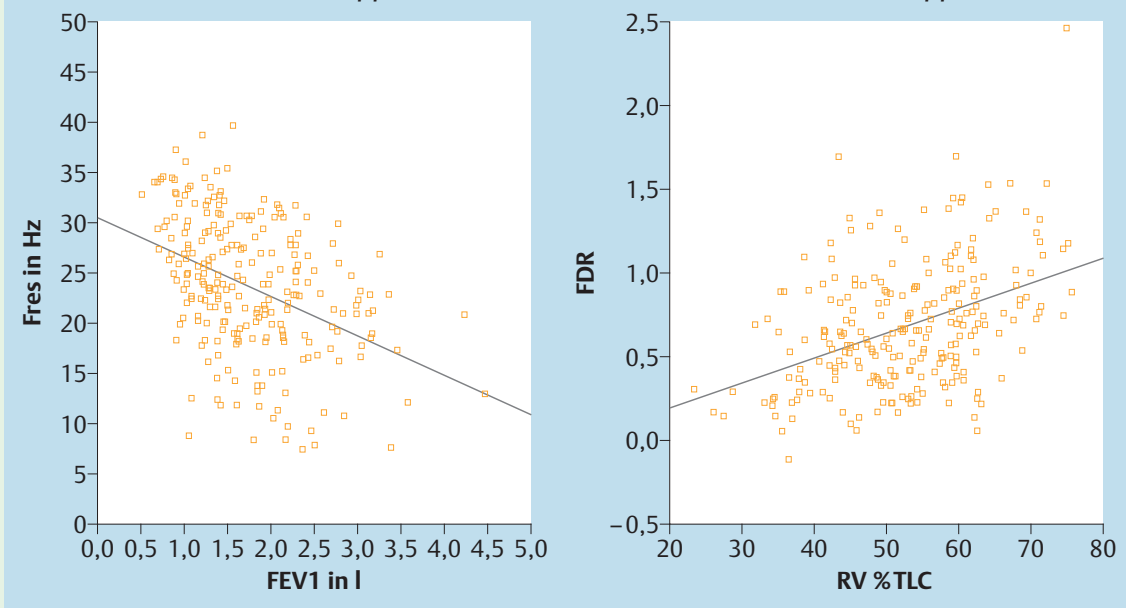

\section{Schweregradeinstufung der Obstruktion}

Aufgrund der mittels Standardmethodik ermittelten Daten wurde das heterogene Patientenspektrum in Untergruppen nach der Schwere der Obstruktion eingeteilt. Einteilungskriterium für die Schweregradabstufungen (ohne Obstruktion=Gruppe 0, leichte Obstruktion = Gruppe 1, mittelgradige Obstruktion = Gruppe 2, schwergradige Obstruktion = Gruppe 3 ) war die in der Bodyplethysmografie gemessene spezifische Resistance ( $\bullet$ Tab. 3). Dabei unterscheiden sich Gruppe 1 vs. 2 ( $p<0,001$ ) sowohl in allen Standardparametern ( Tab. 7) als auch, mit Ausnahme von R20, in den Parametern der IOS ( Tab. 8). Anhand der TLC \%SW, lassen sich beide Gruppen, im Gegensatz zu RV \%TLC und VCin \%SW, nicht signifikant voneinander trennen, was auch bei schwerer Obstruktion (Gruppe 3) auf eine relative Lungenüberblähung schließen lässt. Mit Ausnahme der peripheren bzw. totalen Atemwiderstände R5 und R20 unterscheiden sich die Gruppen 1, 2 und 3 in den übrigen Parametern der IOS deutlich.

Die bezüglich der Frühdiagnostik der Obstruktion besonders relevanten Gruppen 0 und 1, welche sich in der bodyplethysmogra- fischen Resistance signifikant unterscheiden, zeigen interessanterweise keine Differenzen in den pneumotachografischen Parametern (muskuläre Kompensation geringer Veränderungen beim forcierten Messmanöver?). Mittels IOS sind Unterschiede der Gruppen 0 vs. 1 (trotz des geringen Stichprobenumfanges der Gruppe 0 und deutlicher Streuungen einzelner Parameter wie X5) durch die Parameter X5 und Fres bereits erfassbar.

R5 und Zrs zeigen deutliche Überlappungsbereiche zwischen den Gruppen 0 und 1. Obwohl sich die Werte der Widerstandsparameter R5 und R20 der Gruppen 0 vs. 1 statistisch nicht unterscheiden, ist FDR (abgeleitet aus R5 und R20) in der Gruppe 0 signifikant geringer. Bei 9 Patienten der Gruppe 0 ohne bodyplethysmografisch nachweisbare Obstruktion (SRtot \%SW =105 $\pm 10,9$ ) ist mittels Pneumotachografie eine leichte Obstruktion (FEV1 \%VCin $=62 \pm 4,9$, FEV1 \%SW $=69 \pm 10,0$ ) bei exspiratorischer Flussminderung (PEF \%SW $=70 \pm 15,5, \quad M E F 50 \% S W=30 \pm 8,6$, MEF25\%SW = 24 $\pm 8,1$ ) nachweisbar. Bei 6 Patienten der Gruppe 0 lässt sich weder bodyplethysmografisch (SRtot \%SW $=99 \pm 8,4$ ) noch mittels Pneumotachografie (FEV1\%VCin $=76 \pm 4$, FEV1 \%SW 


\begin{tabular}{|c|c|c|c|c|c|c|c|}
\hline $\begin{array}{l}\text { Parameter } \\
\mathrm{MW} / \mathrm{s}\end{array}$ & $\begin{array}{l}\text { Gruppe } 0 \\
\text { ohne } \\
n=15\end{array}$ & 0 vs. 1 & $\begin{array}{l}\text { Gruppe } 1 \\
\text { leicht } \\
n=97\end{array}$ & 1 vs. 2 & $\begin{array}{l}\text { Gruppe } 2 \\
\text { mittel } \\
n=105\end{array}$ & 2 vs. 3 & $\begin{array}{l}\text { Gruppe } 3 \\
\text { schwer } \\
n=27\end{array}$ \\
\hline Alter (J) & $62 / 13,8$ & - & $59 / 14,4$ & * & $64 / 13,0$ & - & $62 / 12,2$ \\
\hline \% männlich & 80 & & 58 & & 62 & & 63 \\
\hline Größe (cm) & $169 / 7,9$ & - & $169 / 7,7$ & - & $169 / 8,8$ & - & $171 / 9,9$ \\
\hline Gewicht (kg) & $73,6 / 9,4$ & - & $75,7 / 15,5$ & - & $75,7 / 16,1$ & - & $79,4 / 20,7$ \\
\hline Rtot (kPa/l/s) & $0,31 / 0,06$ & $* * *$ & $0,45 / 0,10$ & $* * *$ & $0,69 / 0,20$ & $* * *$ & $1,10 / 0,22$ \\
\hline Rtot \% SW & $102 / 20,7$ & $* * *$ & $149 / 32,9$ & $* * *$ & $231 / 66,0$ & $* * *$ & $368 / 73,2$ \\
\hline SRtot \% SW & $103 / 10,0$ & $* * *$ & $158 / 22,9$ & $* * *$ & $281 / 60,3$ & $* * *$ & $536 / 89,1$ \\
\hline FEV1 (I) & $2,21 / 0,68$ & - & $2,06 / 0,68$ & $* * *$ & $1,56 / 0,54$ & $* * *$ & $1,17 / 0,56$ \\
\hline FEV $1 \%$ SW & $75 / 11,8$ & - & $72 / 17,3$ & $* * *$ & $58 / 18,9$ & $* * *$ & $40 / 15,7$ \\
\hline FEV1\% VCin & $68 / 8,5$ & - & $66 / 9,6$ & $* * *$ & $58 / 12,3$ & $* * *$ & $47 / 11,5$ \\
\hline PEF \% SW & $72 / 17,1$ & - & $66 / 19,9$ & $* * *$ & $53 / 19,3$ & $* *$ & $39 / 17,2$ \\
\hline MEF50\% SW & $41 / 17,1$ & - & $40 / 19,5$ & $* * *$ & $26 / 14,7$ & $* * *$ & $16 / 16,1$ \\
\hline MEF $25 \%$ SW & $35 / 15,8$ & - & $32 / 18,0$ & $* * *$ & $22 / 12,2$ & $* * *$ & $13 / 11,3$ \\
\hline TLC \% SW & $94 / 11,5$ & - & $97 / 11,8$ & $* * *$ & $105 / 14,4$ & - & $111 / 15,4$ \\
\hline VCin \% SW & $86 / 12,0$ & - & $84 / 15,7$ & $* * *$ & $77 / 15,8$ & $* *$ & $65 / 14,2$ \\
\hline RV \% TLC & $45 / 7,2$ & - & $47 / 8,8$ & $* * *$ & $56 / 8,3$ & $* * *$ & $64 / 7,8$ \\
\hline
\end{tabular}

Tab. 7 Standardparameter der Lungenfunktion bei verschiedenen Obstruktionsschweregraden bei Ruheatmung, gemessen an SRtot in $\%$ des Sollwertes.

\begin{tabular}{|c|c|c|c|c|c|c|c|}
\hline $\begin{array}{l}\text { Parameter } \\
\mathrm{MW} / \mathrm{s}\end{array}$ & $\begin{array}{l}\text { Gruppe } 0 \\
\text { ohne } \\
n=15\end{array}$ & $0 / 1$ & $\begin{array}{l}\text { Gruppe } 1 \\
\text { leicht } \\
n=97\end{array}$ & $1 / 2$ & $\begin{array}{l}\text { Gruppe } 2 \\
\text { mittel } \\
n=105\end{array}$ & $2 / 3$ & $\begin{array}{l}\text { Gruppe } 3 \\
\text { schwer } \\
n=27\end{array}$ \\
\hline R5 (kPa/l/s) & $0,50 / 0,13$ & - & $0,59 / 0,20$ & $* * *$ & $\mathbf{0 , 7 4} / 0,25$ & - & $0,84 / 0,30$ \\
\hline R5\% SW & $157 / 41,9$ & - & $176 / 65,5$ & $* * *$ & 219/72,7 & - & $250 / 81,4$ \\
\hline $\mathrm{R} 20(\mathrm{kPa} / \mathrm{l} / \mathrm{s})$ & $0,36 / 0,08$ & - & $0,39 / 0,13$ & - & $0,42 / 0,13$ & - & $0,41 / 0,15$ \\
\hline R20\% SW & $130 / 25,8$ & - & $136 / 47,2$ & - & $144 / 43,1$ & - & $141 / 52,9$ \\
\hline FDRa & $0,14 / 0,08$ & $*$ & $0,20 / 0,12$ & $* * *$ & $0,32 / 0,17$ & $* *$ & $0,43 / 0,20$ \\
\hline FDR & $0,38 / 0,20$ & $*$ & $0,51 / 0,26$ & $* * *$ & $0,78 / 0,34$ & $* * *$ & $1,09 / 0,42$ \\
\hline X5 (kPa/l/s) & $-0,16 / 0,08$ & $*$ & $-0,22 / 0,12$ & $* * *$ & $-0,35 / 0,16$ & $* * *$ & $-0,55 / 0,37$ \\
\hline Fres $(\mathrm{Hz})$ & $16,9 / 5,1$ & $*$ & $20,6 / 5,0$ & $* * *$ & $26,1 / 5,9$ & $* *$ & $29,9 / 6,2$ \\
\hline $\mathrm{Zrs}(\mathrm{kPa} / \mathrm{l} / \mathrm{s})$ & $\mathbf{0 , 5 2} / 0,14$ & - & $\mathbf{0 , 6 3 / 0 , 2 2}$ & $* * *$ & $\mathbf{0 , 8 3 / 0 , 2 7}$ & $* *$ & $1,04 / 0,39$ \\
\hline Zrs \% SW & $164 / 45,1$ & - & $188 / 70,5$ & $* * *$ & $243 / 81,3$ & $* *$ & $307 / 104,2$ \\
\hline
\end{tabular}

- = nicht signifikant; * $\mathrm{p}<0,05 ;{ }^{* *} \mathrm{p}<0,01 ;{ }^{* * *} \mathrm{p}<0,001$, Überschreitungen der Sollwerte fettgedruckt.

$=84 \pm 8,9)$ eine Obstruktion erfassen. In der Flussvolumenkurve ist jedoch bereits eine exspiratorische Flussminderung feststellbar (PEF \%SW $=75 \pm 20,5, \mathrm{MEF} 50 \% \mathrm{SW}=58 \pm 11,2$, MEF25 \%SW $=51 \pm 9,5)$. Für beide Populationen der Gruppe 0 zeigen sich impulsoszillometrisch bereits Sollwertüberschreitungen für verschiedene Parameter. Sowohl für die Patienten mit pathologischem $(n=9)$ als auch mit normalem Tiffeneau-Index $(n=6)$ fallen abweichende Mittelwerte besonders für R5 $(156 \pm 43,6 \% \mathrm{SW}$ vs. $157 \pm 43,4 \% \mathrm{SW})$, für $\mathrm{Zrs}(162 \pm 48,8 \% \mathrm{SW}$ vs. $167 \pm 48,1 \% \mathrm{SW})$ und Fres $(16 \pm 5,8 \mathrm{~Hz}$ vs. $18 \pm 4,1 \mathrm{~Hz})$ sowie grenzwertige Befunde für X5 $(-0,15 \pm 0,09 \mathrm{kPa} / \mathrm{l} / \mathrm{s}$ vs. $-0,18 \pm 0,07 \mathrm{kPa} / 1 / \mathrm{s})$ auf (Sollwerte der Gesamtgruppe s. $\bigcirc$ Tab. 5). Die Werte von R20 sind nicht in der Lage, die einzelnen Gruppen voneinander zu trennen. Die aus R5 und R20 abgeleitete Frequenzabhängigkeit der Resistance (FDR), die bezüglich ihres Betrages den Resistancewerten nicht direkt vergleichbar ist, nimmt mit dem Obstruktionsgrad kontinuierlich zu und zeigt signifikante Unterschiede zwischen allen Gruppen. Die Absolutwerte für R5 und Zrs liegen für die Gruppen 0,1 und 2 über denen von Rtot (überschätzte Obstruktion?). Erst bei schweren Obstruktionen liegen die durch IOS gemessenen Werte unter denen für Rtot. Diese für die Oszillationsmethodik typische „Unterschätzung“ schwerer Obstruktionen ist bei Zrs gegenüber R5 geringer ausgeprägt, sodass Zrs vergleichbar zu den anderen komplexeren IOS-Parametern FDR, X5 und Fres signifikante Unterschiede zwischen mittelschwerer und schwerer
Obstruktion abbildet. Insgesamt zeigt der grafische Vergleich $(\bullet$ Abb. 4), dass die IOS-Absolutwerte verschiedene Schweregrade der Obstruktion adäquat abbilden können, wobei typische Unterschiede zur bodyplethysmografischen Resistance besonders in den Gruppen 0 (Überschätzung) und 3 (Unterschätzung) bestehen und die komplexeren IOS-Parameter Zrs, FDRa und X5 sowie Fres gegenüber R5 Vorteile aufweisen. Die IOS-Parameter wie Zrs und X5 streuen insbesondere in Gruppe 3 stärker als die bodyplethysmografische Resistance.

\section{Diskussion}

Die in dieser Studie im Vergleich zu den Widerstands-Messverfahren geringeren quantitativen Sollwertabweichungen der Flussparameter dürften wesentlich durch das Messprinzip und die Mitarbeitsabhängigkeit mitbedingt sein. Die endexspiratorischen Flusswerte zeigen hierbei die deutlichsten Sollwertabweichungen, was auf die Bedeutung der kleinen Atemwege bei obstruktiven Erkrankungen hinweist. Das relative FEV1 mit Bezug auf die Vitalkapazität weist geringere Streuungen gegenüber dem absoluten FEV1-Wert auf, hat aber den Nachteil einer geringeren Sensitivität bei vorliegender Lungenüberblähung mit reduzierter Vitalkapazität als Bezugsgröße. In der Gruppe mit leichtbis mittelschwerer Obstruktion werden aufgrund des relativen 


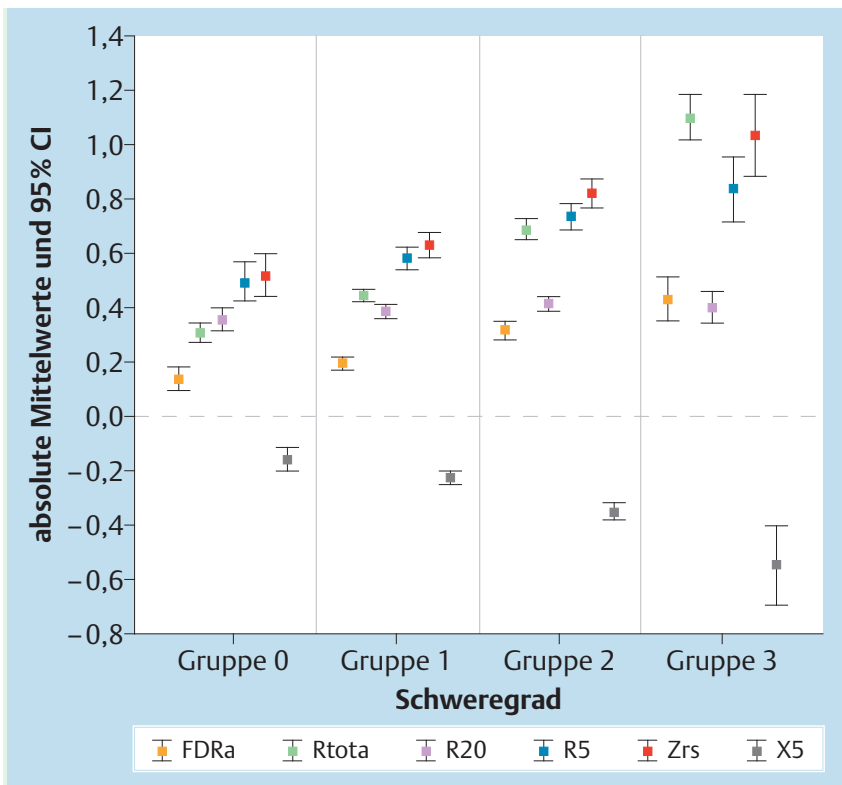

Abb. 4 Vergleich ausgewählter Atemwegswiderstandsparameter (Absolutwerte) bezüglich der Unterscheidung verschiedener Schweregrade der Obstruktion; Gegenüberstellung von Bodyplethysmografie und Impulsoszillometrie.

FEV1 jedoch nur acht von 190 Patienten weniger als obstruktiv eingestuft als aufgrund der absoluten Einsekundenkapazität. Die Parameter FEV1 und Rtot bzw. SRtot gelten als Standard für die Erfassung bronchialer Obstruktionen [57,69-72] und trotz der geringeren Sensitivität wird die hohe Spezifität der Spirometrieparameter als Vorteil gesehen, was sich international auch in der Betonung des FEV1 als Leitparameter in den GOLD-Richtlinien $[57,70]$ und des FEV1/PEF in den GINA-Leitlinien ausdrückt $[11,69,73,74]$. Der erhebliche Anteil von Patienten dieser Untersuchung, bei denen die Obstruktion nur bodyplethysmografisch erkannt wird, spricht gegen die alleinige Anwendung der FEV1Messung, jedoch für die Sensitivität der Resistancemessung A $[25,75]$ und damit den Einsatz von Widerstandsmessverfahren unter Ruheatmung. Unterschiede zwischen den Erkrankungsgruppen COPD und Asthma waren aufgrund des methodischen Untersuchungsansatzes und der Stichprobenchrakteristika nicht Gegenstand der vorliegenden Untersuchung.

Bei der Widerstandsmessung liegt für die Gesamtgruppe dieser Studie R5 in Übereinstimmung mit einer kürzlich veröffentlichten Arbeit gering über Rtot [76]. Dabei wurde der mittlere Raw ebenfalls kleiner gemessen als die oszillometrischen Resistancewerte bei niedrigen Frequenzen als Hinweis darauf, dass die IOS-Resistancewerte in diesen Bereichen gering überschätzt werden. Der Unterschied zwischen R5 (IOS) und Raw (Bodyplethysmografie) war für die Gesamtgruppe jedoch nur gering (im Mittel 0,1 kPa/l/ s), wobei die Abhängigkeit vom Obstruktion-Schweregrad zu beachten ist. Insgesamt liegen die Normabweichungen von Zrs und R5 zwischen denen von SRtot und Rtot, was eine vergleichbare Messempfindlichkeit dieser Parameter für die Gesamtgruppe nahelegt. Besonders häufige und deutliche Normwertüberschreitungen zeigen in unserer Untersuchungsgruppe die bodyplethysmografischen Werte der totalen Resistance mit statistischer Überlegenheit der spezifischen Resistance gegenüber Rtot. Dieser Unterschied lässt sich durch die Berücksichtigung der Lungenüberblähung im Parameter SRtot erklären, da mit höherer Atemmittellage eine mechanische Vordehnung der kleinen Atemwege erfolgt und somit mittels Rtot scheinbar niedrigere Widerstands- werte gemessen werden [28]. Bei der Bewertung von IOS-Obstruktionsparametern in der Schweregradeinstufung werden spirometrische Parameter und bodyplethysmografische Resistancewerte als Referenzverfahren eingesetzt [41, 53, 61, 62, 65, 77 -83]. Unterschiede der bestimmten Messparameter sind bekannt, wobei gering höhere Normwertgrenzen für den oszillometrisch gegenüber dem bodyplethysmografisch gemessenen Atemwegswiderstand angegeben werden [51,61,84-87].

Von den in dieser Untersuchung mittels IOS anhand von FDR, X5 und Fres als leicht obstruktiv eingestuften Patienten der Gruppe 0 wiesen jedoch neun einen pathologischen Tiffeneau-Index auf, während die verbleibenden sechs Patienten bei normalem Index eine deutliche exspiratorische Flussminderung im Sinne einer „small airways disease“ aufwiesen. Somit finden früh obstruktive IOS-Befunde (trotz normaler SRtot) in der Pneumotachografie ein pathologisches Korrelat, was eher für eine hohe Sensitivität als für eine rein methodische Überschätzung spricht. Eine Erhöhung von Rtot resultiert nach bisherigen Erkenntnissen wesentlich aus der Einengung der zentralen Atemwege [31]. Die IOS vermag hingegen, bedingt durch das wenig flussabhängige Messprinzip, Obstruktionen der peripheren Atemwege sensitiver zu erfassen $[59,65]$. Dies birgt jedoch die Gefahr „falsch positiver“ Werte, denen „normale“ bodyplethysmografische Resistancewerte im Sinne einer geringeren Spezifität („Überschätzung leichter Obstruktionen“) gegenüberstehen. Allerdings wäre eine verallgemeinernde Wertung hier nur anhand einer größeren Gruppe 0 unter Einbeziehung von Bronchoprovokation und ggf. exhalativer NO-Messung möglich. Nach dieser Studie sind für eine statistische Differenzierung der Gruppen 0 und 1 die Parameter Reactance (X5), Resonanzfrequenz (Fres) und Frequenzabhängigkeit der Resistance (FDR) besonders geeignet. Die höhere Empfindlichkeit von R5 gegenüber R20 in dieser Studie zeigt die größere Aussagefähigkeit niedrigfrequenter Oszillationsmessungen, die auf der besseren Erfassung der peripheren Atemwege beruhen dürfte.

Beim mittleren Obstruktionsschweregrad stimmen die Absolutwerte von R5 und Impedanz noch gut mit der bodyplethysmografischen Resistance überein, wobei Zrs höhere Werte als R5 ergibt. Bei schwergradigen Obstruktionen zeigt sich eine Unterschätzung des durch die spezifische Resistance (SRtot) beschriebenen Obstruktionsgrades sowohl durch die bodyplethysmografische Resistance (Rtot) als auch durch den IOS-Parameter R5 sehr deutlich, während die Pneumotachografie ausgeprägte Flusseinschränkungen erfasst. Mehrfach fanden sich IOS-Resistancewerte sowohl bei höheren Messfrequenzen als auch mit zunehmender Obstruktion deutlich unter bodyplethysmografischen Werten mit dadurch bedingter Unterschätzung schwerer Obstruktionen $[61,66,76,85,88]$. Die diagnostische Information einer Atemwegsobstruktion geht mit steigendem Grad zunehmend auf die Reactance X mit einer höheren Sensitivität bei niedrigen Messfrequenzen über [89]. Mit dem Kombinationsparameter Impedanz Zrs, der die Komponenten $\mathrm{R}$ und $\mathrm{X}$ einbezieht, ist jedoch eine bessere Einschätzung schwerer Obstruktionen zu erwarten $[61,66]$. Folgerichtig weicht in unserer Untersuchung die Impedanz Zrs bei schwerer Obstruktion wesentlich geringer von Rtot $\mathrm{ab}$ als R5. Zusätzlich zeigt die Frequenzabhängigkeit FDR trotz größerer Streuungen eine bessere Erfassung schwerer Obstruktionen gegenüber R5. In der Subgruppenanalyse zeigt die von der Reactance abhängige Fres, die als empfindlicher für die Charakterisierung einer Obstruktion im Vergleich zur Resistance beschrieben wird $[63,65]$, wie auch X5 im Gegensatz zu R5 signifikante Unterschiede zwischen allen Schweregraden einschließ- 
lich Gruppe 3 mit schwerer Obstruktion. Diese Studie zeigt, dass die hochfrequente oszillometrische Resistance (R20) als Einzelparameter nicht wesentlich zur Schweregradabschätzung der Obstruktion beiträgt, wohl jedoch zur Berechnung der Frequenzabhängigkeit der Resistance. Eine ausreichende Empfindlichkeit für den Obstruktionsnachweis mittels IOS wird dahingegen besonders für niedrige Messfrequenzen gesehen [61, 65, 66, 87, 90 92].

Da neben den beiden Impedanzkomponenten $\mathrm{R}$ und $\mathrm{X}$ diagnostische Informationen über Art und Ausmaß einer Atemwegsobstruktion im gesamten, von der IOS-Methode erfassten Frequenzverlauf vorhanden sind, werden Vorteile von formspezifischen Kennwerten erwartet, welche die Frequenzabhängigkeit von R(f) und X(f) quantitativ charakterisieren. Analog zur klassischen Oszillometrie und in Anlehnung an die Literatur $[60,68]$ wurde deshalb in der vorliegenden Studie die mit stärkerer Obstruktion zunehmende Frequenzabhängigkeit von $\mathrm{R}$ durch den Parameter FDR, welcher bisher kein IOS-Routineparameter ist, quantifiziert. Dieser stellt ein relatives Maß durch die Normierung auf R20 dar, es werden in der Literatur auch absolute Differenzen R5-R20 bzw. R5 - R15 angegeben [53,61]. Ein hoher FDR-Wert charakterisiert somit eine größere Steilheit der frequenzabhängigen Resistancekurve, bedingt durch höhere R5 und/oder niedrigere R20Werte. Diese Frequenzabhängigkeit der Resistance wird als ein guter Marker für den Obstruktionsgrad und als sensitiver Indikator einer frühen Atemwegsobstruktion gesehen [37,66, 83,9395]. Beim Vergleich gesunder und symptomarmer asthmakranker Kinder erwies sich die FDR als die am besten trennende Messgröße [60]. In der vorliegenden Studie weist neben X5 und Fres nur die FDR signifikante Unterschiede zwischen allen ObstruktionsSchweregraden auf und liegt, bezogen auf die Sollwertabweichung, trotz höherer Streuungen in den Gruppen 0 und 3 der Rtot am nächsten. Die Abgrenzung mittelgradiger gegenüber leichtgradiger Obstruktionen war mit allen untersuchten Parametern außer R20 gut möglich. Für die Aussagekraft der komplexeren IOS-Parameter spricht auch deren gegenüber R5 deutlichere Korrelation zu Rtot und auch zu FEV1 in dieser Studie.

Rtot und R5 (IOS) korrelieren in Übereinstimmung mit einer anderen Studie vergleichsweise schwach [76]. Vereinzelt wurden auch höhere Korrelationen, z. B. zwischen Ros bei $6 \mathrm{~Hz}$ (FOT) und Raw beschrieben [63]. Die vorliegende Studie zeigt trotz insgesamt niedriger Korrelationskoeffizienten eine Korrelation zur Pneumotachografie für X5, Fres und FDR, wobei die Korrelation von Fres zu FEV1 gut mit den Ergebnissen bei älteren Patienten übereinstimmt [96], die in einer anderen Studie für Fres beschriebenen Korrelationskoeffizienten jedoch unterschreitet [97]. Bei Kindern mit Asthma war die Korrelation von X5 geringer als die von R5 [98].

Die relativ geringen Korrelationen zwischen oszillometrischer und bodyplethysmografischer Resistance bei COPD werden durch messmethodische Unterschiede erklärt [66,85,87]. Insgesamt sind jedoch Korrelationen der IOS zur Bodyplethysmografie deutlicher als zur Pneumotachografie/Spirometrie. In einer Studie mit einer gemischten obstruktiv-restriktiven Patientengruppe finden sich die höchsten Korrelationen von Rtot zu Fres, Zrs, X5 und zu R5, wobei der mittlere Rtot der Gesamtgruppe mit $0,47 \mathrm{kPa} / \mathrm{l} / \mathrm{s}$ niedriger als in der vorliegenden Studie war [62]. Für die Widerstandswerte bei höheren Frequenzen fanden sich ebenfalls geringere bzw. keine signifikanten Korrelationen zum bodyplethysmografischen Widerstand. Korrelationen zu den exspiratorischen Flusswerten (MEF) wurden nur für R5 und für Zrs berichtet [62]. Zwischen FEV1 und Parametern der multifrequenten Oszillome- trie wie R8, X8 und Fres wurden Korrelationskoeffizienten $<0,3$ beschrieben [96,99]. Bei einer Untersuchung an 216 Asthmapatienten lagen die Korrelationen von R5 und Zrs zum FEV1 bei - 0,49 [97], bei jugendlichen Asthmatikern fanden sich enge Korrelationen zwischen FEV1 und R5, X5 sowie R5 - R15 [53].

Betrachtet man bei der Charakterisierung der Obstruktion in der Gesamtgruppe die Empfindlichkeit der Standardparameter, gemessen an der Häufigkeit normabweichender Befunde, so schneidet SRtot am besten ab, gefolgt von Rtot, was die Bedeutung der im deutschsprachigen Raum gut etablierten und international wieder aufgewerteten Bodyplethysmografie belegt. Unter den IOS-Parametern erwiesen sich FDR, Fres und Zrs als besonders sensitiv. Deren Normwertüberschreitungen lagen signifikant über denen der Widerstände R5 und R20. Während R5 und Zrs Variationskoeffizienten vergleichbar der Standardmethodik aufweisen, ist die Streubreite der Reactance X5 und der Frequenzabhängigkeit FDR (vergleichbar zu den exspiratorischen Flusswerten MEF25 bzw. MEF50) recht hoch, was deren Aussagekraft einschränkt. Dennoch wird die Frequenzabhängigkeit der Resistancewerte (FDR) mit zunehmender Obstruktion als brauchbarer Parameter hervorgehoben und durch die vorliegende Studie bestätigt [61,62]. Für eine Messung schwergradiger Obstruktionen mittels IOS sind nach diesen Daten Reactance (X5), Resonanzfrequenz (Fres), Impedanz (Zrs) und Frequenzabhängigkeit der Resistance (FDR) geeigneter als R5, um Nachteile der klassischen Oszillometrie zu vermeiden.

Zusammenfassend vermögen die Pneumotachografie, die Bodyplethysmografie und die IOS Kriterien einer Obstruktion in den entsprechenden Leitparametern deutlich aufzuzeigen, wobei bei einem großen Patientenanteil die Widerstands- und komplexeren IOS-Parameter deutlich von den Sollwerten abweichen, was für eine insgesamt gute Sensitivität spricht. Unter den IOS-Parametern zeigen die FDR und die Fres, gefolgt von X5, R5 und der aus beiden Parametern abgeleiteten Impedanz Zrs eine der bodyplethysmografischen Resistance vergleichbare, gegenüber den Flussparametern jedoch höhere Empfindlichkeit. Die insgesamt relativ schwachen Korrelationen der IOS-Parameter zur Pneumotachografie und Bodyplethysmografie sind aus den Unterschieden der physikalischen Messprinzipien zu erklären [59,62, 71,99-101]. Die IOS hat nach der vorliegenden Untersuchung aufgrund ihrer hohen Empfindlichkeit und der sehr guten Handhabbarkeit das Potenzial für eine aussagekräftige, wenig aufwendige Vorfeldmethode, mit der sich beginnende, klinisch jedoch noch nicht manifeste Obstruktionen sensitiver als allein mit der Pneumotachografie nachweisen lassen. Besonders die Parameter X5 und Fres sowie abgeleitet Zrs erfassen zusätzlich erhöhte Blindwiderstände, die auf ein verändertes bronchopulmonales Trägheits- und Elastizitätsverhalten und somit auf strukturelle Wandeigenschaften hinweisen. Die Nachteile oszillometrischer Messverfahren bei der Erfassung schwerer Obstruktionen können durch die abgeleiteten komplexeren Parameter bei höheren Messwertstreuungen zum Teil ausgeglichen werden. Die Schwächen der klassischen Oszillometrie mit Überschätzung leichter und Unterschätzung schwerer Obstruktionen sind bei Verwendung geeigneter und komplexer IOS-Parameter deutlich geringer ausgeprägt, sodass die IOS bei Interpretation komplexer Parameter als moderne Weiterentwicklung mit praktischen Vorteilen zu betrachten ist. Die Autoren empfehlen deshalb den Einsatz der IOS besonders als Ergänzung zur Pneumotachografie/Spirometrie bei fehlender Bodyplethysmografie und bei kooperationsgeminderten Patienten sowie zur Früh- und Screeningdiagnostik bronchialer Obstruktionen. 


\section{Literatur}

1 Vogel J, Smith HJ. Entwicklungsstand der Impulsoszillometrie 2003. Pneumologie 2003; 57: 763-764

2 Aronsson D, Tufvesson E, Ankerst J et al. Allergic rhinitis with hyper-responsiveness differ from asthma in degree of peripheral obstruction during metacholine challenge test. Clin Physiol Funct Imaging 2008; 28: $81-85$

3 Park JW, Lee YW, Jung YH et al. Impulse oscillometry for estimation of airway obstruction and bronchodilation in adults with mild obstructive asthma. Ann Allergy Asthma Immunol 2007; 98: 546 - 552

4 Oppenheimer BW, Goldring RM, Herberg ME et al. Distal airway function in symptomatic subjects with normal spirometry following World Trade Center dust exposure. Chest 2007; 132: 1275-1282

5 Nguyen TU, Diong B, Nazeran H et al. A study of IOS data using two mead-related models of respiratory impedance. Conf Proc IEEE Eng Med Biol Soc 2007; 2007: 1078 - 1081

6 Al-Mutairi SS, Sharma PN, Al-Alawi A et al. Impulse oscillometry: an alternative modality to the conventional pulmonary function test to categorise obstructive pulmonary disorders. Clin Exp Med 2007; 7: $56-64$

7 Singh D, Tal-Singer R, Faiferman I et al. Plethysmography and impulse oscillometry assessment of tiotropium and ipratropium bromide; a randomized, double-blind, placebo-controlled, cross-over study in healthy subjects. Br J Clin Pharmacol 2006; 61: 398 - 404

8 Houghton CM, Woodcock AA, Singh D. A comparison of plethysmography, spirometry and oscillometry for assessing the pulmonary effects of inhaled ipratropium bromide in healthy subjects and patients with asthma. Br J Clin Pharmacol 2005; 59: 152 - 159

9 Shiota S, Katoh M, Fujii M et al. Predictive equations and the reliability of the impulse oscillatory system in Japanese adult subjects. Respirology 2005; 10: $310-315$

10 ATS, American Thoracic Society. Standards for the diagnosis and care of patients with chronic obstructive pulmonary disease (COPD) and asthma. Am J Respir Crit Care Med 1995; 152: 77-120

11 Gillissen A. Die chronisch obstruktive Lungenerkrankung. Bremen: UNI-MED Science, 2003

12 Lange P, Parner J, Vestbo P et al. A 15-year follow-up study of ventilatory function in adults with asthma. N Engl J Med 1998; 339: $1194-$ 1200

13 Nolte D. Lungenfunktionsdiagnostische Basisverfahren. Prax Klin Pneumol 1987; 41: 474-476

14 Reichel G. Standard und Praxis der funktionellen Diagnostik chronischer Lungenerkrankungen. Atemwegs-Lungenkrankh 1993; 19: $67-72$

15 Saetta M, Finkelstein R, Cosio MG. Morphological and cellular basis for airflow limitation in smokers. Eur Respir J 1994; 7: 1505-1515

16 Thurlbeck WM. Pathology of chronic airflow obstruction. Chest 1990; 97: $6 s-10 s$

17 O'Donnell DE, Sanii R, Anthonisen NR et al. Effect of dynamic airway compression on breathing pattern and respiratory sensation in severe chronic obstructive pulmonary disease. Am Rev Respir Dis 1987; 135: $912-918$

18 Smith HR, Irvin CG, Cherniack RM. The utility of spirometry in the diagnosis of reversible airways obstruction. Chest 1992; 101: 15771581

19 Becklake MR. Chronic airflow limitation: its relationship to work in dusty occupations. Chest 1985; 88: 608-617

20 Macklem PT. Obstruction in small airways-a challenge to medicine. Am J Med 1972; 52: 721 - 724

21 Petro W, Konietzko N. Atlas der pulmonalen Funktionsdiagnostik. Darmstadt: Steinkopff Verlag, 1989

22 Löllgen $H$. Kardiopulmonale Funktionsdiagnostik. 3. Auflage. Nürnberg: Novartis Pharma GmbH, 2000

23 Murray AB, Ferguson AC. A comparison of spirometric measurements in allergen bronchial challenge testing. Clin Allergy 1981; 11: 87-93

24 Orehek J. Measurement of airway hyperresponsiveness in man. Eur J Resp Dis 1982; 117: 42-61

25 Seidel A. Atemstoßtest, Atemgrenzwert, Resistance und Fluß-Volumenkurve - Möglichkeiten einer umfassenden Charakterisierung der Atemwegsobstruktion und der Atemreserve. Pneumologie 1998; 52: $437-438$

26 Smidt $U$, Schulz V, Ferlinz R. Physiologie und Pathophysiologie. In: Ferlinz R (Hrsg). Pneumologie in Klinik und Praxis. Stuttgart, New York: Georg Thieme Verlag, 1994: 25-66
27 Nolte D. Einführung zum IOS-Workshop Bad Reichenhall. In: Vogel J, Nolte D, Smith HJ (Hrsg). Workshop Impuls-Oszillometrie Bad Reichenhall. München-Deisenhofen: Dustri-Verlag Dr. Karl Feistle, 1995

28 Ulmer WT, Schläfke ME. Die Lungenfunktion: State-of-the-Art und neue Ergebnisse. Pneumologie 2003; 57: 400-401

29 Dubois AB, Bothelo SY, Comroe JH. A new method for measuring airway resistance in man using a bodyplethysmograph: Values in normal subjects and in patients with respiratory disease. J Clin Invest 1956; 35: $327-335$

30 Ciappi G, De Benedetto F, D'llario F et al. Chronobiological aspects of bronchial tone. Chronobiologia 1982; 9: 163-172

31 Schnellbächer $F$. Lungenfunktionsprüfungen mit der einfachen und erweiterten Oszilloresistometrie, Schriftenreihe Zentralblatt für $\mathrm{Ar}$ beitsmedizin, Arbeitschutz, Prophylaxe und Ergonomie 8. Heidelberg: Dr. Curt Haefner Verlag, 1981

32 Gonsior E, Krüger M, Meier-Sydow J. Die Durchführung inhalativer Antigen-Provokationsproben mit Hilfe der Ganzkörperplethysmografie. Acta aller 1976; 31: 283-296

33 Kanengiser S, Dozor AJ. Forced expiratory maneuvers in children aged 3 to 5 years. Pediatr Pulmonol 1994; 18: $144-149$

34 Sly PD, Robertson CF. A review of pulmonary function testing in children. J Asthma 1990; 27: 137-147

35 Dubois $A B$, Brody AW, Lewis DH, Burgess BF. Oscillation mechanics of lung and chest in men. J Appl Physiol 1956; 8: 587-594

36 Smith HJ, Vogel J, Arnold J et al. Impuls-Oszillometrie in der Früherkennung obstruktiver Atemwegserkrankungen. Sonderausgabe IOS. Höchberg: Firma Jaeger, 1997

37 Brochard L, Pelle G, de Palmas J et al. Density and frequency dependence of resistance in early airway obstruction. Am Rev Respir Dis 1987; 135: 579-584

38 Cuijpers CE, Wesseling GJ, Kessels AG et al. Low diagnostic value of respiratory impedance measurements in children. Eur Respir J 1997; 10: $88-93$

39 Glaser T, Pleger E, Müller E et al. Erprobungsbericht zur polyfrequenten Oszilloresistometrie mit dem Gerät custo vit. Pneumologie 1989; 43: $369-375$

40 Peslin R, Felicio da Silva J, Duvivier Cet al. Respiratory mechanics studied by forced oscillations during artificial ventilation. Eur Respir J 1993; 6: $772-784$

41 Bisgaard H, Klug B. Lung function measurement in awake young children. Eur Respir J 1995; 8: 2067 - 2075

42 Frey $U$, Silverman $M$, Kraemer R et al. High-frequency Respiratory Impedance Measured by Forced-Oscillation Technique in Infants. Am J Respir Crit Care Med 1998; 158: 363-370

43 Hall GL, Hantos Z, Wildhaber JH et al. Methacholine responsiveness in infants assessed with low frequency forced oscillation and forced expiration techniques. Thorax 2001; 56: $42-47$

44 Malmberg LP, Mäkelä MJ, Mattila PS et al. Exercise-induced changes in respiratory impedance in young wheezy children and nonatopic controls. Pediatr Pulmonol. 2008; 43: 538-44

45 Song TW, Kim KW, Kim ES et al. Correlation between spirometry and impulse oscillometry in children with asthma. Acta Paediatr. 2008; 97: $51-54$

46 Nieto A, Pamies $R$, Oliver $F$ et al. Montelukast improves pulmonary function measured by impulse oscillometry in children with asthma (Mio study). Respir Med 2006; 100: 1180-1185

47 Tomalak W, Radliński J, Pawlik J et al. Impulse oscillometry vs. body plethysmography in assessing respiratory resistance in children. Pediatr Pulmonol 2006; 41: 50-54

48 Frei J, Jutla J, Kramer $G$ et al. Impulse oscillometry: reference values in children 100 to $150 \mathrm{~cm}$ in height and 3 to 10 years of age. Chest 2005; 128: $1266-1273$

49 Olaguíbel JM, Alvarez-Puebla MJ, Anda M et al. Comparative analysis of the bronchodilator response measured by impulse oscillometry (IOS), spirometry and body plethysmography in asthmatic children. J Investig Allergol Clin Immunol 2005; 15: 102 - 106

50 Marotta A, Klinnert MD, Price MR et al. Impulse oscillometry provides an effective measure of lung dysfunction in 4-year-old children at risk for persistent asthma. J Allergy Clin Immunol 2003; 112: 317-322

51 Vogel J, Smidt U. Impulsoszillometrie. Analyse der Atemmechanik in Ambulanz und Klinik, Epidemiologie und experimenteller Forschung. Frankfurt/M: pmi Verlagsgruppe, 1994

52 van de Woestijne KP, Desager KN, Duiverman EJ et al. Recommendations for measurement of respiratory input impedance by means of the forced oscillation method. Eur Respir Rev 1994; 4: 235-237 
53 Goldmann MD, Carter R, Klein R et al. Within - and between - day variability of respiratory impedance, using impulse oscillometry in adolescent asthmatics. Pediatr Pulmonol 2002; 34: 312 - 319

54 ATS. American Thoracic Society. Lung function testing: selecting of reference values and interpretive strategies. Am Rev Respir Dis 1991; 144: $1202-1218$

55 ERS. European Respiratory Society. Standarized lung function testing Lung volumes and forced ventilatory flows. update. Eur Respir J 1993; 6: $5-40$

56 Deutsche Atemwegsliga. Durchführung von Lungenfunktionsprüfungen in der Praxis. Pneumologie 1994; 48: 292 - 295

57 GOLD. Global strategy for the diagnosis, management and prevention of chronic obstructive pulmonary disease (updated 2007). National institutes of health. www.goldcopd.com, 2007

58 Schmidt $W$. Angewandte Lungenfunktionsprüfung. Eine Einführung für Praxis und Klinik. 4. Auflage. München: Dustri-Verlag, 1990

59 Wouters EFM. Total respiratory impedance measurement by forced oscillations: a non-invasive method to assess bronchial response in occupational medicine. Exp Lung Res 1990; 16: 25 - 40

60 Buhr W, Landser F, Reimnitz P et al. Lungenfunktionsuntersuchungen an gesunden und symptomarmen asthmakranken Kindern im Alter von 5 bis 16 Jahren. Atemwegs-Lungenkrankh 1987; 13: 370 - 374

61 Müller E, Harzendorf E, Merker $G$ et al. Theoretische und experimentelle Untersuchungen zur diagnostischen Relevanz und Vergleichbarkeit oszillationsmechanischer Parameter. Pneumologie 1989; 43: $339-347$

62 Schmidt $U$. Untersuchungen zum Stellenwert der Impulsoszillometrie in der Diagnostik restriktiver Lungenfunktionsstörungen. Medizinische Dissertation Berlin: 1998

63 Fichter J, Wilkens JH, Fabel H. Die oszillatorische Messung des Atemwiderstands mit dem custo vit im Vergleich zum Oszillaire und zur Bodyplethysmografie. Pneumologie 1989; 43: 382 - 386

64 Fisher $A B$, DuBois $A B$, Hyde RW. Evaluation of the forced oscillation technique for the determination of the resistance to breathing. J Clin Invest 1968; 47: 2045-2057

65 Kohlhäufl M, Brand P, Scheuch G et al. Impulse oscillometry in healthy nonsmokers and asymptomatic smokers: effects of bronchial challenge with methacholine. J Aerosol Med 2001; 14: 1-12

66 Landser FJ, Nagels J, Demedts $M$ et al. A new method to determine frequency charakteristics of the respiratory system. J Appl Physiol 1976; 41: $101-106$

67 Michaelson ED, Grassmann ED, Peters WR. Pulmonary mechanics by spectral analysis of forced random noise. J Clin Invest 1975; 56: $1210-1230$

68 Pasker HG, Mertens I, Clement J et al. Normal values of total respiratory input resistance and reactance for adult men and women. Eur Respir Rev 1994; 4: 134-137

69 Deutsche Atemwegsliga. Leitlinie zur Diagnostik und Therapie der COPD. Kurzfassung. Stuttgart: Thieme Verlag, 2002

70 GOLD. Global initiative for chronic Obstructive Lung Disease. www. goldcopd.com, 2001

71 van Noord JA, Smeets J, Clement J et al. Assessment of reversibility of airflow obstruction. Am J Crit Care Med 1994; 150: 551 - 554

72 Wise RA, Connett J, Kurnow K et al. Selection of spirometric measurements in a clinical trial, the Lung Health Study. Am J Respir Crit Care Med 1995; 151: 675-681

73 Gillissen A, Wirtz H, Hoheisel G. Paradigmenwechsel in der Therapieempfehlung des Asthma bronchiale - New Perspectives in GINA Asthma Guideline 2006. Med Klin 2007; 102: 399-403

74 Pauwels RA, Buist AS, Calverley PM et al., GOLD Scientific Committee. Global strategy for the diagnosis, management, and prevention of chronic obstructive pulmonary disease. NHLBI/WHO Global Initiative for Chronic Obstructive Lung Disease (GOLD) Workshop summary. Am J Respir Crit Care Med 2001; 163: 1256-1276

75 Taube C, Lehnigk B, Paasch $\mathrm{K}$ et al. Factor analysis of changes in dyspnea and lung function parameters after bronchodilatation in chronic obstructive pulmonary disease. Am J Respir Crit Care Med 2000; 162 : $216-220$

76 Hellinckx J, Cauberghs M, De Boeck Ket al. Evaluation of impulse oscillation system: comparison with forced oscillation technique and body plethysmography. Eur Respir J 2001; 18: 564- 570

77 Klug B, Bisgaard $H$. Measurement of lung function in awake 2-4-year-old asthmatic children during methacholine challenge and acute asthma: a comparison of the impulse oscillation technique, the inter- rupter technique, and transcutaneous measurement of oxygen versus whole-body plethysmography. Pediatr Pulmonol 1996; 21: 290-300

78 Kaminsky DA, Irvin CG. New insights from lung function. Curr Opin Allergy Clin Immunol 2001; 1: 205-209

79 Malmberg LP, Pelkonen A, Poussa T et al. Determinants of respiratory system input impedance and bronchodilator response in healthy Finnish preschool children. Clin Physiol Funct Imaging 2002; 22: 64-71

80 Ortiz G, Menendez $R$. The effects of inhaled albuterol and salmeterol in 2- to 5-year old asthmatic children as measured by impulse oscillometry. J Asthma 2002; 39: 531 - 536

81 Nielsen KG, Bisgaard H. Discriminative capacity of bronchodilator response measured with three different lung function techniques in asthmatic and healthy children aged 2 to 5 years. Am J Respir Crit Care Med 2001; 164: 554-559

82 Wesseling GJ, Vanderhoven-Augustin IM, Wouters EF. Forced oscillation technique and spirometry in cold air provocation tests. Thorax 1993; 48: $254-259$

83 van Noord JA, Clement J, van de Woestijne KP et al. Total respiratory resistance and reactance in patients with asthma, chronic bronchitis and emphysema. Am Rev Respir Dis 1991; 143: 922-927

84 Nussbaum E, Galant SP. Measurement of total respiratory resistance in children by a modified forced oscillation method. Pediatr Res 1984 18: $139-145$

85 Petro W, Loytved G, Korn Vet al. Inhalativer bronchialer Provokationstest - diagnostische Aussagekraft verschiedener Funktionsmethoden (Spirografie, Pneumotachografie, Bodyplethysmografie, Oszillometrie). Prax Klin Pneumol 1983; 37: 85 - 90

86 Smith HJ, Vogel J. Praktische Umsetzung der Impuls-Oszillometrie Stand 2003. Pneumologie 2003; 57: 765

87 Nolte D, Korn V. Oszillatorische Messung des Atemwiderstandes. München: Dustri-Verlag, 1979

88 Förster E, Berger D, Nolte D. Vergleichsmessungen des Atemwiderstandes mit der Oszillationsmethode und mit der Bodyplethysmografie. Verh Dtsch Ges Inn Med 1978; 84: 392 - 395

89 Pleger E, Müller E, Wilke A et al. Kann die oszillatorische Einschätzung des Atemwiderstandes chronisch obstruktiver Patienten durch den Einsatz eines verdoppelten Referenzwiderstandes verbessert werden? Z Erkr Atmungsorgane 1985; 165: 143-148

90 Buhr W, Jörres R, Berdel D et al. Correspondence between forced oscillation and body plethysmography during bronchoprovokation with carbachol in children. Pediatr Pulmonol 1990; 8: 280-288

91 Sixt $R$, Aronsson PH, Solymar $L$. The forced oscillation technique in children - with special reference to the use of low frequencies. Eur Respir Rev 1991; 1: $222-225$

92 Wuthe H, Uhrner R, Kleckow $M$ et al. Verbesserung der Atemwiderstandsmessung durch Senkung der oszillatorischen Frequenz. Prax Klin Pneumol 1988; 42: 790-792

93 Clement J, Landser FJ, van de Woestijne KP. Total resistance and reactance in patients with respiratory complaints with and without airway obstruction. Chest 1983; 83: 215-220

94 Delacourt C, Lorino H, Herve-Guillot $M$ et al. Use of the forced oscillation technique to assess airway obstruction and reversibility in children. Am J Crit Care Med 2000; 161: 730 - 736

95 Kjeldgaard JM, Hyde RW, Speers DM et al. Frequency dependence of total respiratory resistance in early airway disease. Am Rev Respir Dis 1976; 114 : 501 - 508

96 Janssens JP, Nguyen MC, Herrmann FR et al. Diagnostic value of respiratory impedance measurements in elderly subjects. Respir Med 2001; 95: 415 - 422

97 Kim CW, Kim JS, Park JW et al. Clinical applications for forced oscillation techniques (FOT) in patients with bronchial asthma. Korean J Intern Med 2001; 16: 80 - 86

98 Vink GR, Arets HG, van der Laag J et al. Impulse oscillometry: a measure for airway obstruction. Pediatr Pulmonol 2003; 35: 214-219

99 Wesseling GJ, Wouters EF. Respiratory impedance measurements in a dose-response study of isocapnic hyperventilation with cold air. Respiration 1992; 59: 259-264

100 Delacourt C, Lorino H, Fuhrmann C et al. Comparision of the forced oscillation technique and the interrupter technique for assessing airway obstruction and its reversibility in children. Am J Respir Crit Care Med 2001; 164: 965 - 972

101 Klein G. Kontinuierliche Messungen des Atemwiderstands (Ros, Ru) bei bronchialen Provokations- und Spasmolysetests. Atemwegs-Lungenkrankh 1995; 21: 199-205 